\title{
Comparative value of transthoracic and transoesophageal echocardiography before balloon dilatation of the mitral valve
}

Martyn R Thomas, Mark J Monaghan, David W Smyth, Jennie M Metcalfe, David E Jewitt

\begin{abstract}
Objective-To assess the relative merits of transthoracic and transoesophageal echocardiography before balloon dilatation of the mitral valve.

Design-Transthoracic and transoesophageal echocardiograms were prospectively performed in 35 patients being considered for balloon dilatation of the mitral valve. Echocardiograms were analysed for image quality, the assessment of valve morphology, the detection of left atrial thrombus, and the assessment of mitral regurgitation and other valvar pathology.
\end{abstract}

Patients-35 consecutive patients with symptomatic dominant mitral stenosis.

Interventions-30 eventually underwent balloon dilatation of the mitral valve by the Inoue technique. Five patients had mitral valve replacement.

Main outcome measures-Echocardiographic and surgical detection of left atrial thrombus and successful, uncomplicated balloon dilatation of the mitral valve.

Results-Left atrial thrombus was detected in 1/35 patients by transthoracic studies compared with $6 / 35$ from transoesophageal studies. Otherwise both techniques gave comparable results. Thrombus was confirmed at mitral valve replacement in five patients. Successful dilatation of the mitral valve was performed in 30 patients.

Conclusions-Transthoracic echocardiography is a useful screening procedure but transoesophageal echocardiography is mandatory before balloon dilatation of the mitral valve for the detection of left atrial thrombus.

Department of Cardiology, King's College Hospital, Denmark Hill, London M R Thomas $M \mathrm{~J}$ Monaghan D W Smyth J M Metcalfe D E Jewitt

Correspondence to Dr D E Jewitt, Department of Cardiology, King's College Hospital, King's College Hospital, SE5 9RS.

Accepted for publication 22 April 1992.
(Br Heart J 1992;68:493-7)

Transvenous balloon dilatation of the mitral valve is now well established as an alternative to surgery in a selected group of patients with symptomatic dominant mitral stenosis. ${ }^{1}$ Various techniques have been used, but all transvenous methods involve manipulation of at least one guide wire and balloon within the left atrium before balloon inflation across the mitral valve. ${ }^{2-5}$ Widely recognised contraindications to the procedure include left atrial thrombus, significant mitral regurgitation, excessive valvar or subvalvar calcification, and significant pathology in other valves such as aortic regurgitation. ${ }^{6}$ Echocardiography plays a vital part in assessing the suitability of patients for balloon dilatation of the mitral valve ${ }^{67} \mathrm{It}$ has been our policy for some time to carry out both transthoracic and transoesophageal echocardiography before dilatation of the mitral valve. The aim of this study was to evaluate the relative merits of the two techniques before balloon dilatation.

\section{Patients and methods}

PATIENTS

Thirty five consecutive patients who were being considered for balloon dilatation of the mitral valve were studied. Patients had been referred from within and outside our institution on the basis of clinical history, clinical examination, and baseline investigations including a transthoracic echocardiogram. The group consisted of 20 women and 15 men. The mean age was 62 (range 22-78) years. Twenty four patients were in atrial fibrillation and 11 in sinus rhythm. Thirty two of the 35 patients were receiving warfarin and all these patients had a prothrombin time of $>1.5$ times that of controls. Five patients had a history of arterial embolic events. Two had had transient ischaemic attacks, one a cerebrovascular accident, and two a femoral embolus.

BALLOON DILATATION OF THE MITRAL VALVE

The Inoue system was used for all cases in this study. The technique has been described in detail elsewhere. ${ }^{2}$ Briefly, a safety J guide wire is positioned within the left atrium after a standard Brockenbrough interatrial septal puncture. The Inoue balloon is then positioned within the left atrium before being guided by flow across the mitral valve for subsequent inflation. With this technique no guide wires are positioned within the left ventricular cavity.

\section{ECHOCARDIOGRAPHY}

All echocardiograms carried out during this study were performed by one of three operators experienced in both transthoracic and transoesophageal echocardiography.

\section{Transthoracic echocardiography}

Transthoracic echocardiography was performed with a Hewlett-Packard 77020 PhasedArray Scanner with a $2 \cdot 5-\mathrm{MHZ}$ transducer. 
Pulsed wave, continuous, and colour flow Doppler were used in all studies.

Transoesophageal echocardiography

Transoesophageal echocardiography was performed with the same Hewlett-Packard system and a $5 \mathrm{MHZ}$ single plane oesophageal transducer with colour flow Doppler.

Each patient underwent transthoracic and transoesophageal echocardiography on the same day, generally immediately before the proposed valve dilatation and always within one month of balloon dilatation of the mitral valve.

\section{Results}

All echocardiograms were analysed independently by two experienced people blinded to the subsequent clinical course of the patient. In the event of disagreement a third observer was used and the result determined by a consensus.

The variables analysed were: image quality; valvar morphology and mitral valve area; left atrial thrombus; mitral regurgitation; other significant lesions-for example, pulmonary hypertension and other valvar lesions.

\section{IMAGE QUALITY}

Some subjectivity in the assessment of image quality is unavoidable. Image quality from each study was scored on a scale of 1 to 5,1 being a very poor image and 5 high quality definition. ${ }^{8}$ An aggregate score of 6 or above (by the two operators carrying out the analysis) was regarded as optimal imaging while any score below this was regarded as suboptimal. Imaging was suboptimal in 6/35 transthoracic echocardiographic images. In all cases this was due to a respiratory artefact and in none of these cases could the mitral valve area be reliably planimetered in the short axis view. Image quality for transoesophageal echocardiography was suboptimal in 4/35. Invariably this was due to a greatly enlarged left atrium that caused displacement of the transducer and created difficulty in adequately aligning the left atrium, mitral valve, and left ventricle (fig 1).

\section{VALVE MORPHOLOGY AND MITRAL VALVE AREA}

An echocardiographic score before balloon dilatation (as used by others) was used to assess valve morphology. ${ }^{7}$ With this system scores of 0 to 4 are assigned to leaflet mobility, thickening, calcification, and subvalvar thickening; higher scores represent increasing morphological abnormalities. Patients with a score of 8 or less are usually excellent candidates for dilatation whereas those with a score of 10 or more have increased complications and a lower success rate. $^{6}$ There was no statistical difference in the mean (SD) echocardiographic score for the two groups. This was $7 \cdot 7(1 \cdot 8)$ for transthoracic echocardiography and $7 \cdot 3(1.6)$ for transoesophageal echocardiography. In five patients, however, leaflet calcification was limited to the leaflet tips on transoesophageal studies whereas calcification had seemed more extensive during transthoracic studies. Figure 2 shows the ease with which valvar and sub-

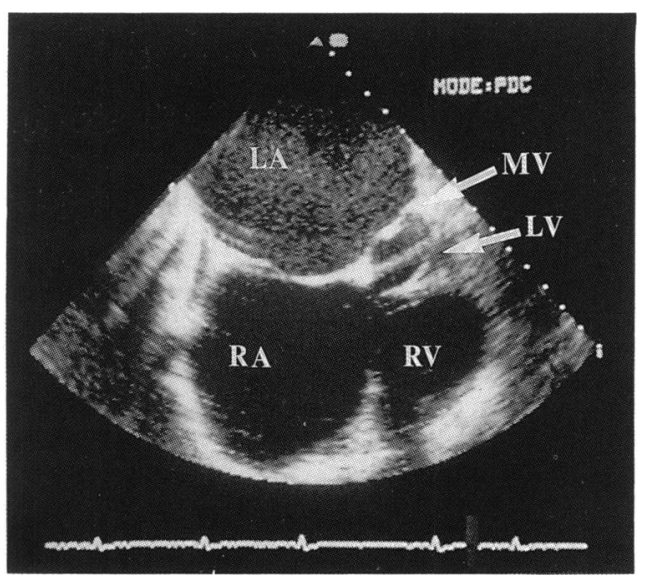

Figure 1 Transoesophageal four chamber image shows the difficulties in imaging the mitral valve when there is a greatly enlarged left atrium. Morphological assessment of the mitral valve ( $M V$ ) and alignment of the left atrium the mitral valve $(M A), M V$, and left ventricle ( $L V$ ) for colour flow studies is obviously unsatisfactory. This is caused by displacement of the transducer leading to horizontal rather than vertical alignment of the right and left heart. $R A$, right atrium; $R V$, right ventricle.

valvar calcification can be assessed during transoesophageal studies.

Mitral valve area, whether assessed by the pressure half time method ${ }^{9}$ or by planimetry, could only be assessed by transthoracic echocardiography. Mean mitral valve area before dilatation was $1.03(0.3) \mathrm{cm}^{2}$ by pressure half time $(\mathrm{n}=35)$ and $1 \cdot 1(0 \cdot 3) \mathrm{cm}^{2}$ by planimetry $(n=29)$. Commisural fusion could also only be assessed by transthoracic echocardiography; as for mitral area this was because a reliable short axis view across the mitral valve with the single plane oesophageal transducer was difficult to obtain. The transthoracic short axis view remains an important image before dilatation as it provides a baseline from which changes may be measured after serial balloon inflations during the procedure.

\section{LEFT ATRIAL THROMBUS}

Thrombus was identified in 1/35 from transthoracic echocardiography compared with $6 / 35$

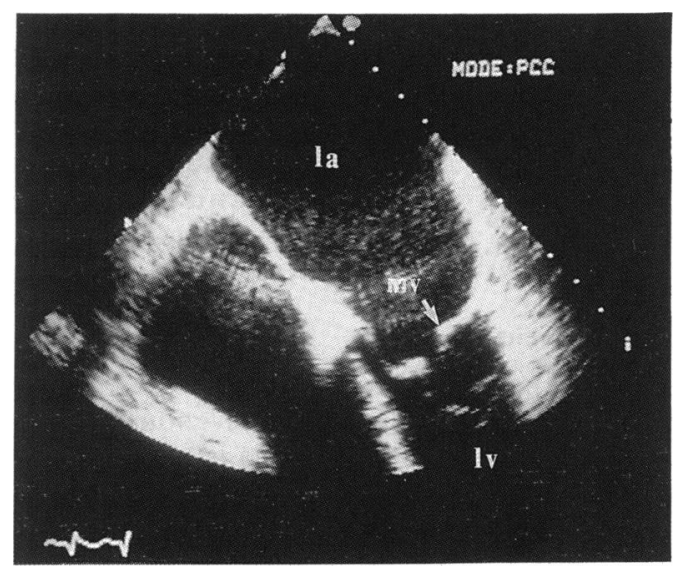

Figure 2 More usual image obtained in the transoesophageal four chamber view compared with figure 1. The left and right heart now lie in a vertical plane and the ease with which mitral valve (mv) valvar, and subvalvular calcification can be assessed. Alignment of the left atrium (la), mv, and left ventricle (lv) is also ideal for colour flow Doppler studies. 
Figure 3 Example of left atrial thrombus ( $L A T)$ seen during

transoesophageal

echocardiography. No

thrombus was seen during

the transthoracic study in

this case. The image shows

$L A T$ within the body of

the left atrium ( $L A$ ) in

the basal short axis view.

There is associated

spontaneous echo contrast within the body of the $L A$.

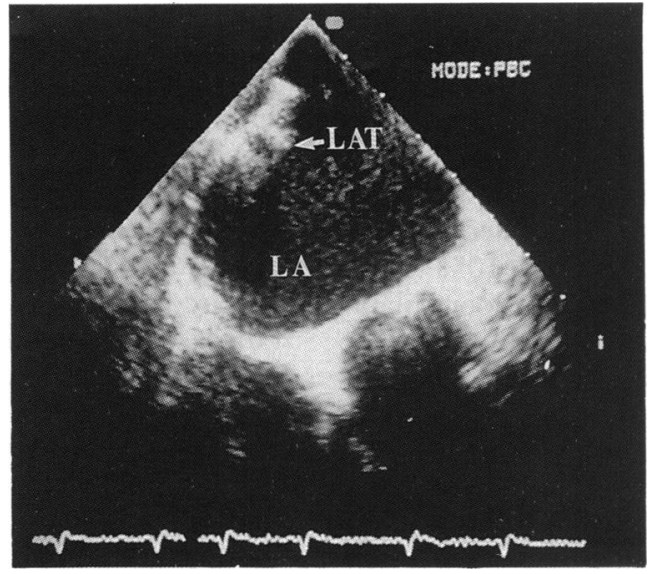

from transoesophageal echocardiography. During transoesophageal studies this was localised to the body of the left atrium in four patients, to the left atrial appendage in one patient, and to both sites in a further patient. Figure 3 shows an example of left atrial thrombus seen during transoesophageal echocardiography. No thrombus was seen during the transthoracic study in this patient. All six patients in whom left atrial thrombus was seen were in atrial fibrillation and receiving anticoagulation treatment.

Three of the six patients with left atrial thrombus had a history of arterial emboli (one cerebrovascular accident, one transient ischaemic attack, and one femoral embolus). Two further patients with a similar history (one transient ischaemic attack and one femoral embolus) had no left atrial thrombus identified. One patient who had organised thrombus within the left atrial appendage, and who was not considered to be fit for mitral valve replacement, did proceed to balloon dilatation of the mitral valve. Mitral valve dilatation was cancelled in the other five patients with left atrial thrombus and they subsequently underwent mitral valve replacement.

Transoesophageal echocardiographic studies alone therefore led to the cancellation of four cases (thrombus seen in one patient during transthoracic studies) who would have proceeded to dilatation of the mitral valve on the basis of the transthoracic study alone. Left atrial thrombus was confirmed in all five patients who underwent mitral valve replacement.

\section{MITRAL REGURGITATION}

Transthoracic echocardiography with continuous and colour flow Doppler allowed mitral regurgitation to be graded on the basis of the maximum distance from the valve orifice attained by the regurgitant signal and on the area covered by the regurgitant signal. ${ }^{10}$ The grading could also be modified by the width of the colour flow jet at the valve orifice, the intensity and timing of the continuous wave Doppler signal, and the proximetry of the jet to a left atrial wall. A similar form of this grading system, as validated by others, was also used for transoesophageal studies. ${ }^{11}$ Analysis of the pul- sed wave Doppler signal of pulmonary venous flow was also used in the assessment of mitral regurgitation during transoesophageal studies.

With this grading system $16 / 35$ patients had mild mitral regurgitation on transthoracic echocardiography. Transoesophageal echocardiography did not alter the grading in any of these patients. A further 10 patients had minimal mitral regurgitation with transoesophageal echocardiography. Transoesophageal echocardiography was particularly useful in assessing eccentric mitral regurgitation, when the ability to sample pulmonary venous flow by pulsed Doppler echocardiography provided important additional information. It is recognised that under circumstances in which regurgitant jets impinge on the atrial wall colour flow mapping alone may underestimate the degree of mitral regurgitation. ${ }^{12}$

Mitral regurgitation during subsequent left ventriculography was graded by the method of Sellers et al..$^{13}$ Thirty four of the 35 patients had grade $1+$ mitral regurgitation or less, while one patient had $2+$ mitral regurgitation.

ASSESSMENT OF OTHER IMPORTANT LESIONS Pulmonary hypertension

From transthoracic echocardiography with continuous wave Doppler 12/35 patients had tricuspid regurgitation and an adequate Doppler envelope for the estimation of systolic pulmonary artery pressures.

\section{Other valvar lesions}

Eight patients had mild aortic valve disease, which could be quantitatively assessed by transthoracic echocardiography with continuous wave Doppler. Pulsed wave Doppler showing no reversed flow in the descending aorta could also be used in the assessment of aortic regurgitation. ${ }^{14}$

In our experience, the alignment of the colour flow Doppler jets of both tricuspid and aortic valve disease is difficult with the single plane transoesophageal probe.

\section{CLINICAL OUTCOME}

Thirty of the 35 patients went on to successful balloon dilatation of the mitral valve. Mean mitral valve area, calculated by planimetry when possible, after the procedure was 1.91 $(0.38) \mathrm{cm}^{2}$, and $27(90 \%)$ patients had an area after dilatation $>1.5 \mathrm{~cm}^{2}$, which has traditionally been classed as an optimal result. No patient developed $>2+$ mitral regurgitation, and no patient experienced an embolic event during the procedure.

Five patients underwent mitral valve replacement and in all these patients left atrial thrombus was confirmed at surgery.

\section{Discussion}

Transvenous balloon dilatation of the mitral valve provides an attractive alternative to surgery in patients with mitral stenosis. However, the procedure is not without risk; arterial embolus is the most catastrophic of the potential complications of the procedure. The 
incidence of left atrial thrombus, diagnosed by transoesophageal echocardiography, in this study was $17 \%$ and is in line with previous reports of $15 \%-22 \%$ in two large series of patients with mitral valve disease. ${ }^{1516}$ Two previous studies have used transoesophageal echocardiography to report an incidence of left atrial thrombus of $26 \%(5 / 19)$ and $10 \%(2 / 20)$ in patients being considered for balloon dilatation of the mitral valve. ${ }^{17} 18$ Our study is the largest reported to date in English publications and the first to report the experience of a United Kingdom centre. It also compares all the echocardiographic data obtained before dilatation with the transthoracic and the single plane transoesophageal approach.

All transvenous valve dilatation techniques, whether with the flow guided Inoue balloon or the wire guided single and double balloons, require some guide wire and balloon manipulation within the left atrium with the risk of dislodging a thrombus. Before the availability of transoesophageal echocardiography, transthoracic echocardiography was the investigation of choice in assessing the suitability of patients for mitral valve dilatation. This imaging technique, however, is known to be unreliable in detecting thrombus within the body of the left atrium, and the left atrial appendage is usually not seen. Vahanian $e t$ al, in a series of 212 patients being considered for mitral valve dilatation, excluded only one patient, because of the detection of left atrial thrombus by transthoracic echocardiography. ${ }^{19}$ The reported incidence, to date, of arterial emboli during balloon dilatation of the mitral valve is $2 \%$ $4 \% .^{19-21}$

Transoesophageal echocardiography now provides a sensitive means of interrogating the left atrium and left atrial appendage. The procedure, however, is semi-invasive and involves a variable amount of discomfort to the patient. Because of this, clear clinical benefit to the patient should be shown before it is introduced into routine practice before mitral valve dilatation. In this study transoesophageal echocardiography alone led to a change in the clinical management of the patient in six $(17 \%)$ patients. In four patients valve dilatation was cancelled because of evidence of left atrial thrombus, whereas in two patients valve dilatation was allowed to proceed when no left atrial thrombus was seen despite a clear history of arterial emboli.

There were no embolic episodes during balloon dilatation of the mitral valve in this series of patients. Before the introduction of routine predilatation transoesophageal echocardiography 33 patients underwent mitral valve dilatation at our hospital (all performed by the same operator). Three patients in this group had transient ischaemic attacks during the procedure. Comparison of the two groups (those with and those without predilatation transoesophageal echocardiography) is, however, difficult because: (a) the first 27 cases were performed by the over the wire technique with single or double balloons. The potential for embolic episodes may be higher in these patients because of increased wire and balloon manipulation in the left atrium and the longer inflation to deflation times of these balloons. All arterial emboli occurred in this group of patients; $(b)$ the learning curve of the operator may have increased the complication rate in this group.

Apart from the assessment of left atrial thrombus, transthoracic echocardiography appeared to compare favourably with the transoesophageal approach. Indeed transthoracic studies had a number of advantages over the single plane transoesophageal images especially in terms of the assessment of mitral valve area and commissural anatomy, and in enabling the monitoring of the results of serial balloon inflations during the procedure. The excellent correlation between mitral regurgitation assessed by echocardiography and left ventricular angiography may represent an under referral of patients with anything other than very mild mitral regurgitation by the transthoracic route.

\section{STUDY LIMITATIONS}

A single plane transoesophageal system without the facility for on line continuous wave Doppler examination was used for the transoesophageal studies in this series. The newer biplane and multiplane transducers may well have a number of advantages over this system and should allow better images of the left atrium and mitral valve apparatus and the assessment of mitral valve area. These advantages, however, are still undergoing clinical evaluation and the oesophageal transducers are not yet widely available.

A definite reduction in arterial emboli, due to the introduction of transoesophageal echocardiography, cannot be claimed as the two groups undergoing mitral valve dilatation (those with, and those without transoesophageal echocardiography) are not strictly comparable. We have, however, had no embolic events during balloon dilatation of the mitral valve since the introduction of the Inoue technique combined with the routine transoesophageal assessment of patients before valve dilatation.

\section{Conclusion}

Transthoracic and transoesophageal echocardiography give complimentary information before balloon dilatation of the mitral valve. Transthoracic studies are useful as a screening investigation in the process of assessing the suitability of patients for balloon dilatation of the mitral valve and adequately evaluates mitral regurgitation, valve morphology, mitral valve area, and the presence of other valvar lesions. The transthoracic approach also supplies important baseline images and measurements that can then be followed up after serial balloon inflations during the procedure. Transthoracic echocardiography, however, will fail to diagnose important left atrial thrombus in a significant number of patients and, therefore, transoesophageal echocardiography is mandatory before balloon dilatation of the mitral valve to identify these high risk patients. With the introduction of this technique into routine clinical practice the risk of dislodging 
left atrial thrombus during mitral valve dilatation should be reduced.

1 Nishimura RA, Holmes DR, Reeder GS. Percutaneous balloon valvuloplasty. Mayo Clin Proc 1990;65:198-220.

2 Inoue K, Owaki T, Nakamura T, Kitamura F, Miyamoto N. Clinical application of transvenous mitral commissurotomy by a new balloon catheter. $J$ Thorac Cardiovasc Surg 1984;87:394-402.

3 Lock JE, Khalilullah M, Shrivasta S, Bahl V, Keane JF. Percutaneous catheter commissurotomy in rheumatic mitral stenosis. N Engl J Med 1985;313:1515-8.

4 Al-Zaibag MA, Kasab SA, Ribeiro P, Fagih MR. Percutaneous double balloon mitral valvotomy in rheumatic mitral stenosis. Lancet 1986;i:757-61.

5 Meier B, Friedli B, Von Segesser L. Valvuloplasty: technical aspects, congenital heart disease. Herz 1988;13:1-13.

aspects, congenital heart disease. Herz 1988;13:1-13.
6 Block PC. Who is suitable for percutaneous balloon mitral valvotomy? Int J Cardiol 1988;20:9-14.
valve

7 Wilkins GT, Weyman AE, Abascal VM, Block PC, Palacios IF. Percutaneous balloon dilatation of the mitral valve: an analysis of the echocardiographic variables related to outcome and the mechanism of dilatation. Br Heart $J$ 1988;60:299-308.

8 National Health Service in Scotland. Comparative value of imaging and Doppler ultrasound systems for examination of the heart. Edinburgh: A Watt, Common services agency, 1991 .

9 Hatle L, Angelsen B, Tromsdal A. Non-invasive assessment of atrioventricular pressure half-time by Doppler of atrioventricular pressure half-time
ultrasound. Circulation 1979;60:1096-104

10 Miyatake K, Izumi S, Okamoto M, et al. Semiquantitative grading of severity of mitral regurgitation by real-time two-dimensional doppler flow imaging technique. $J A m$ Coll Cardiol 1986;7:82-8.
11 Sheikh KH, Bengtson JR, Rankin JS, de Bruijn NP, Kisslo J. Intraoperative transesophageal Doppler color flow imaging used to guide patient selection and operative treatment of ischemic mitral regurgitation. Circulation 1991;84:594-604.

12 Chen C, Thomas JD, Anconina J, et al. Impact of impinging wall jet on color Doppler quantification of mitral regurwall jet on color Doppler quantification

13 Sellers RD, Levy MJ, Amplatz K, Lillehei CW. Left retrograde cardioangiography in acquired cardiac disease. Am J Cardiol 1964;14:437-47.

14 Hatle L, Angelsen B. Doppler ultrasound in cardiology: physical principals and clinical applications. 2nd ed Philadelphia: Lea and Febiger, 1985:154-62.

15 Daniel WG, Nelleson U, Schroder E, et al. Left atrial spontaneous echo contrast in mitral valve disease: an indicator for an increased thromboembolic risk. J Am Coll Cardiol 1988;11:1204-11.

16 Black IW, Hopkins AP, Lee LC, Walsh WF. Left atrial spontaneous echo contrast: a clinical and echocardiographic analysis. J Am Coll Cardiol 1991;18:398-404.

17 Kronzon I, Tunick PA, Glassman E, Slater J, Schwinger M, Freedberg RS. Transesophageal echocardiography to detect atrial clots in candidates for transeptal mitral balloon valvuloplasty. J Am Coll Cardiol 1990;16:1320-2.

18 Manning WJ, Reis GJ, Douglas PS. Use of transoesophageal echocardiography to detect left atrial thrombi before percutaneous balloon dilatation of the mitral valve: a prospective study. Br Heart J 1992;67:170-3.

19 Vahanian A, Michel PL, Cormier B, et al. Results of percutaneous mitral commissurotomy in 200 patients. $\mathrm{Am}$ J Cardiol 1989;63:847-52.

20 McKay RG. Balloon valvuloplasty for treating pulmonary, mitral, and aortic valve stenosis. Am J Cardiol 1988;61: mitral,

21 Palacios IF, Block PC, Wilkins GT, Weyman AE. Followup of patients undergoing percutaneous mitral balloon valvotomy. Circulation 1989;79:573-9. 